\section{¿Cómo abordan su aprendizaje los estudiantes de medicina autónomos? Una aproximación cualitativa}

\author{
CAROLINA MÁRQUEZ U. a, EDUARDO FASCE H., \\ JAVIERA ORTEGA B. ${ }^{b}$, CAROLINA BUSTAMANTE D. ${ }^{c}$, \\ CRISTHIAN PÉREZ V. , PILAR IBÁÑEZ G. e, LILIANA ORTIZ M., \\ CAMILA ESPINOZA P. ${ }^{\mathrm{f}}$, NANCY BASTÍAS V. ${ }^{\mathrm{g}}$
}

\section{Learning strategies of autonomous medical students}

Background: Understanding how autonomous students are capable of regulating their own learning process is essential to develop self-directed teaching methods. Aim: To understand how self-directed medical students approach learning in medical schools at University of Concepción, Chile. Material and Methods: A qualitative and descriptive study, performed according to Grounded Theory guidelines, following Strauss \& Corbin was performed. Twenty medical students were selected by the maximum variation sampling method. The data collection technique was carried out by a semi-structured thematic interview. Students were interviewed by researchers after an informed consent procedure. Data were analyzed by the open coding method using Atlas-ti 7.5.2 software. Results: Self-directed learners were characterized by being good planners and managing their time correctly. Students performed a diligent selection of contents to study based on reliable literature sources, theoretical relevance and type of evaluation. They also emphasized the discussion of clinical cases, where theoretical contents can be applied. This modality allows them to gain a global view of theoretical contents, to verbalize knowledge and to obtain a learning feedback. Conclusions: The learning process of autonomous students is intentional and planned.

(Rev Med Chile 2015; 143: 1579-1584)

Key words: Learning; Students, medical; Qualitative research.
Departamento de Educación Médica, Facultad de Medicina, Universidad de Concepción,

Concepción, Chile.

aBioquímica, Magíster en Educación Médica para las Ciencias de la Salud.

bPsicóloga, Magíster en Ciencias de la Educación.

'Enfermera, Magíster en

Enfermería.

dPsicólogo, Magíster en Psicología.

'Matrona, Magíster en

Educación.

fPsicóloga, Magíster en Psicología Educacional.

${ }^{9}$ Enfermera, Magíster en Educación Médica para las Ciencias de la Salud.

Recibido el 2 de julio 2015; aceptado el 27 de octubre de 2015

Financiado por Proyecto FONDECYT Regular № 1140654.

Correspondencia a: Carolina Márquez U. Departamento de Educación Médica, Facultad de Medicina, Universidad de Concepción. Barrio Universitario $s / n$, Concepción, Chile. Teléfono: 412204932 cmarquezu@udec.cl
$\mathrm{D}$ iversos estudios en escuelas de Medicina y Ciencias de la Salud sostienen la importancia de desarrollar y evaluar el aprendizaje autodirigido durante los procesos formativos de sus estudiantes ${ }^{1-3}$. La expansión del conocimiento disciplinar ha provocado que las carreras de Medicina y las de Ciencias de la Salud transformen sus currículos tradicionales a aquellos que promueven e integren el aprendizaje independiente. Las habilidades de aprendizaje autodirigido, vistas como prerrequisitos de capacitación continua, son particularmente importantes en este campo donde el conocimiento avanza y cambia continuamente ${ }^{2,3}$.

El aprendizaje autodirigido ha sido referido como un proceso de aprendizaje de carácter estratégico y autoreflexivo, que ocurre de manera grupal o independiente, ya sea en actividades curriculares en el aula o extracurriculares fuera de ella, con la participación directa o indirecta del profesor ${ }^{4}$. En este proceso, el alumno toma la iniciativa, con o sin la ayuda de otros, para diagnosticar sus necesidades de aprendizaje, formular sus metas, identificar materiales y recursos humanos, 
implementar y elegir las estrategias adecuadas y evaluar los resultados de su propio aprendizaje $\mathrm{e}^{5-8}$.

Investigaciones en estudiantes de medicina chilenos han demostrado que los estudiantes que son autodirigidos valoran más la autodirección y la seguridad, utilizando estilos de aprendizaje de tipo teórico y estrategias de aprendizaje metódicas y orientadas al aprendizaje profundo. ${ }^{9}$ Por otra parte, los estudios se han enfocado en evaluar los factores intervinientes, evidenciando la existencia de múltiples factores tanto de los alumnos, docentes y el sistema educativo que influyen en el aprendizaje autodirigido y que para desarrollar esta habilidad se requieren estrategias de tipo cognitivo, metacognitivas, motivaciones e incluso afectivas ${ }^{1}$.

Pese a lo anterior, el carácter cuantitativo de los estudios realizados ha permitido identificar los factores asociados al aprendizaje autodirigido, pero no el proceso a través del cual se conectan con éste. Considerando lo anterior, surge la necesidad de realizar estudios en Chile orientados a dilucidar y comprender cómo los estudiantes procesan la información que estudian y qué criterios utilizan para escoger determinadas técnicas de estudio. Asimismo, es de suma importancia el realizar estudios de tipo cualitativos que brindan la posibilidad de abordar el fenómeno de manera procesual y ahondar en dimensiones emergentes que puedan contribuir a comprender este fenómeno en estudiantes de las escuelas de medicina en Chile.

En vista de lo anterior, el presente estudio busca caracterizar cómo estudian los alumnos autodirigidos en la carrera de Medicina de una universidad tradicional de Chile.

\section{Material y Método}

Este estudio es una investigación cualitativa basada en la Teoría Fundamentada según los lineamientos de Strauss y Corbin ${ }^{10}$. Los participantes fueron 20 estudiantes de cuarto y quinto año de la carrera de Medicina, escogidos mediante muestreo de máxima variación como arranque muestral, identificando un grupo heterogéneo de estudiantes según su desempeño académico, autonomía y sus características personales ${ }^{11}$. El muestreo teórico identificó a los sujetos que mejor respondían a las necesidades del muestreo analítico para alcanzar la saturación de datos ${ }^{12}$, previo proceso de consentimiento informado. Los participantes tenían una edad media de 23,3 años $(\mathrm{DE}=1,8)$ de los cuales $45 \%(\mathrm{n}=9)$ eran mujeres y $55 \%(n=11)$ eran hombres.

Se utilizó la entrevista semi-estructurada, como técnica de recolección de datos ${ }^{12}$. El análisis de datos se realizó a partir del método de comparación constante de Strauss y Corbin hasta el nivel de codificación abierta, el cual permite identificar y describir categorías a partir del contenido señalado por los sujetos participantes, empleando el programa Atlas-ti 7.5.2. El estudio fue aprobado por el Comité de Ética de la Universidad ejecutora, y visado por el Comité Asesor de Bioética de CONICYT.

\section{Resultados}

A partir de los datos analizados, se obtuvo en total 2.210 códigos en vivo, lo que permitió ser fiel al discurso inicial de los participantes y contribuyó a comprender las temáticas generales que se generaron a partir de las entrevistas semi-estructuradas. En el nivel de codificación abierta $^{10}$ emergieron dos categorías referentes a cómo estudian los aprendices autodirigidos: Planificación del estudio y técnicas de análisis en el proceso de aprendizaje.

\section{Planificación del proceso de aprendizaje en estudiantes autodirigidos}

Los estudiantes autodirigidos se caracterizan por planificar su proceso de estudio, a través de metas de estudio previamente establecidas. Con respecto a esto, se menciona que es fundamental ser ordenado al momento de estudiar y para esto surge la necesidad de establecer objetivos claros a corto y largo plazo.

"En cuanto al estudio me gusta la metodología, trato de ser ordenado y plantearme objetivos a corto plazo principalmente, ya que los objetivos a largo plazo están un poco más marcados por lo que dicta la malla" [Entrevistado 7; 50 año, hombre, 23 años].

Otra forma de organización mencionada por los estudiantes fue aquella basada en la calendarización, a través de la cual define un sistema de estudio, con las clases y con los certámenes. Esto permite ir clasificando temáticas día a día.

"Intento, hacer una planificación del semestre; 
tener como todas las pruebas, los certámenes, los trabajos; organizar las materias; tenerlo como, no sé, tenerlo como en el computador, cada uno en su carpeta, tenerlo ordenado" [Entrevistado 14; $4^{\circ}$ año, mujer, 22 años].

Por otro lado, algunos estudiantes mencionan estrategias de cómo seleccionar y estructurar la materia a estudiar. Un primer paso es seleccionar la materia que debe estudiar a largo plazo y luego enfocar el estudio en aquello que debe saber pronto, de esta forma estará activa en la memoria a corto plazo.

"Primero me armo el esquema, llego: 'ya, para mañana tengo que estudiarme estas dos cosas, ¿cuál estudio primero?', generalmente estudio primero la que es para más largo plazo, para que se me quede, y la segunda es para mañana, para que se me quede por que la memoria está más fresca” [Entrevistado 7; $5^{\circ}$ año, hombre, 23 años].

Por otro lado, otra opción es clasificar días por temas de estudio, que luego son resumidos en hojas o cartulinas para ser diferenciados.

"Trato de separar por días los temas y (...) en hojas aparte o en cartulinas para diferenciar los temas. Porque en el cuaderno todas las hojas son iguales. Cuando uno hace cartulina es distinto, con los colores queda más, ayuda harto" [Entrevistado $5 ; 5^{\circ}$ año, hombre, 24 años].

\section{Técnicas de análisis en el proceso de aprendizaje}

Los alumnos, cuando ya poseen los insumos que utilizarán para estudiar, comienzan un proceso en el cual analizan la información con que disponen. Este proceso se caracteriza por una lectura más consciente de los contenidos, pudiendo comenzar a seleccionar esta información. En primer lugar, este análisis se realiza mediante la lectura de la materia, existiendo dos tipos de lectura: la que constituye una primera aproximación y la que se realiza en mayor profundidad.

"Mi técnica de estudio es leer una vez, como dar una pincelada para ver de qué se trata, después leo de nuevo y subrayo" [Entrevistado 10; $5^{\circ}$ año, mujer, 30 años].

Ahora, para priorizar el contenido de estudio un criterio fundamental es evaluar la relevancia del contenido, según si es frecuentemente revisado o visto durante las distintas instancias académicas.
"En Medicina Interna los certámenes igual eran, por ejemplo, en Cardiología, todo lo de Cardiología o de repente de insuficiencia cardíaca, después de las hipertensiones secundarias (...) Por ejemplo, yo le doy prioridad a las que encuentro que hemos visto más (...) y no sé dejo dos de lado" [Entrevistado 1; $4^{\circ}$ año, mujer, 25 años].

Otro modo de determinar que un contenido es relevante, se relaciona con cuánto se aborda durante las clases, de modo que un contenido ampliamente cubierto por el docente en la clase se considera relevante. Asimismo, los alumnos pueden utilizar la información que poseen alumnos mayores, que ya han cursado la asignatura, para determinar qué contenidos son los relevantes, en el marco de las evaluaciones correspondientes.

"Bueno, priorizo los temas que nos dicen en clases, los que hemos visto, los que veo en el hospital $y$ los que preguntando en otros años 'oye, si, ¿sabes? Que esto no preguntan tanto', 'ah, ya, perfecto, no preguntan tanto"' [Entrevistado 1; $4^{\circ}$ año, mujer, 25 años].

Finalmente, los alumnos pueden determinar que un tópico es relevante según la prevalencia de determinadas enfermedades en la población. Así, al momento de tener que estudiar varias patologías, consideran más importantes aquellas que se presentan más frecuentemente en casos clínicos.

Un segundo criterio utilizado es el de la utilidad práctica del contenido, es decir, el cómo se pueden abordar diversas situaciones clínicas o cómo manejar a los pacientes que ven durante estas instancias.

"No sé, ahí uno como que sabe cuáles son los temas como más importantes, o lo más importante de cada tema. Por ejemplo, yo creo que es como saber bien el manejo, eso al final es súper importante, la clínica, qué exámenes tomar, qué no tomar" [Entrevistado $8 ; 4^{\circ}$ año, hombre, 23 años].

Esto puede incluso conllevar a que los alumnos filtren información de un texto, por ejemplo, en función de su aplicabilidad en el ámbito clínico. Por ende, frente a grandes cantidades de información, priorizarán aquellos datos que se refieran al manejo de pacientes y a la práctica clínica en general.

"si el apunte es largo, uno ya sabe, ya 'esto es importante y esto no'. Un poco como visto más a la práctica, más que antes. Porque cuando uno entra, 
al principio, uno se lee todo el apunte, como que todo es importante. Después uno va viendo en sala, ya, 'esto sirve en la vida real, esto no' (...). Yo creo que todos están como adoptando eso, lo que sirve como en el aprendizaje real, como a la hora de ejercer la carrera" [Entrevistado 9; 4 año, hombre, 23 años].

Finalmente, los alumnos declaran utilizar las evaluaciones como criterios para determinar qué van a priorizar durante su estudio. En este sentido, enfocan y dirigen el estudio hacia aquellos contenidos que serán evaluados, en función de obtener un mejor rendimiento. Esto incluso puede significar que los alumnos no estudiarán contenidos que no serán evaluados.

"Por ejemplo, me acuerdo en oftalmología, los tumores, esos, todo el mundo decía 'no, nunca lo preguntan'. Ah, entonces uno le deja para el final y ese me lo leí no más, no me lo estudié" [Entrevistado 1; $4^{\circ}$ año, mujer, 25 años].

Algunos de los alumnos declaran sólo utilizar este criterio para priorizar los contenidos que estudian, enfatizando la importancia que tiene el estudiar contenidos que asegurarán buenos resultados en el ramo por sobre cualquier otro criterio. El siguiente entrevistado detalla la importancia que reviste para él estudiar contenidos que le asegurarán la aprobación del ramo, y dejar una buena impresión en el docente.

“... si lo llevo al plano académico, es como la importancia que tiene tal cosa como para pasar el ramo o la percepción que puede generar esa cosa en el docente evaluador, por ejemplo, quizá no vale tanto, pero si uno no lo hace bien y deja una mala impresión, igual pa mi es fome, uno trata igual de responder de cierta forma" [Entrevistado $8 ; 4^{\circ}$ año, hombre, 23 años].

\section{Discusión}

Favorecer las habilidades de estudio independiente es considerado como un objetivo primordial en la formación médica de pregrado ${ }^{13,14}$, por cuanto se ha comprobado su correlación con un mayor dominio cognitivo ${ }^{15} \mathrm{y}$ su favorable impacto en el desarrollo de competencias clínicas ${ }^{16}$.

La mayor parte de los estudios publicados han estado orientados a establecer los niveles de predisposición al aprendizaje autodirigido ${ }^{17}$, cambios experimentados a través de los años de estudio $^{18,19}$, su relación con diferentes métodos instruccionales ${ }^{20-23}$ y su efecto sobre la motivación para realizar investigación ${ }^{24}$.

En una investigación cualitativa realizada por Tolsgaard en un grupo piloto de estudiantes de medicina daneses de $4^{\circ}$ y $5^{\circ}$ año ${ }^{21}$, se comprobaron positivos efectos sobre el compromiso en la solución de problemas clínicos y el establecimiento de metas mediante la utilización de fichas de encuentros clínicos. Sin embargo, su aplicación en la totalidad de los estudiantes no logró apreciaciones favorables debido a la falta de soporte por parte de los docentes.

La evaluación longitudinal de la predisposición al aprendizaje autodirigido en estudiantes de medicina canadienses demostró una reducción de esta variable, medida con la escala de Guglielmino, entre los años 2006 y $2010^{19}$. Pero la parte cualitativa del estudio permitió identificar aspectos vinculados al aprendizaje autodirigido: necesidad de disponer de tiempo para abordar el estudio y los sistemas de evaluación como condicionante de la forma de estudiar, resultados que coinciden con factores identificados en nuestra investigación.

Estudios recientes presentan modelos comprensivos que involucran dimensiones como la autogestión, el automonitoreo y la motivación ${ }^{6,25}$. Se entiende la autogestión como la capacidad para diagnosticar necesidades de aprendizaje, plantearse metas propias y gestionar recursos y apoyo disponible; el automonitoreo como lo que engloba procesos cognitivos y metacognitivos para construir nuevo conocimientos a partir del andamiaje con conocimientos previos y, finalmente, la motivación como factores externos e internos que influyen en las creencias del alumnos sobre su propio éxito en el aprendizaje ${ }^{6,26}$; sin embargo, es necesario comprender cómo se va desarrollando el aprendizaje autodirigido y qué hace que un estudiante pueda ser más o menos autodirigido que otro.

En relación a los resultados obtenidos en este estudio, los estudiantes de medicina utilizan diversas técnicas para facilitar su proceso de aprendizaje. Un alumno autodirigido establecerá metas para planificar su estudio y utilizará ciertos criterios para seleccionar los contenidos de enseñanza que aprenderá. Ahora bien, el establecimiento de metas a corto plazo y largo plazo, y la selección de contenidos pudiera estar condicionada por el 
tipo de evaluaciones que tienen los estudiantes. Desde este punto de vista, es fundamental analizar qué función está cumpliendo la evaluación en el proceso de aprendizaje de los estudiantes. Si un alumno se guía por las evaluaciones se pudiera interpretar que es más dirigido externamente que autodirigido, sin embargo, cuando el estudiante escoge ser menos o más autodirigido son otros los factores que están influyendo en el desarrollo de éste en la formación profesional.

A partir de los resultados obtenidos en este estudio, se sugiere la necesidad de continuar investigando la forma en que los estudiantes autodirigidos regulan su aprendizaje, en función de diversos factores que podrían influir en sus comportamientos y que van desde el docente hasta el propio ambiente educativo, basándonos en la evidencia que teorías sociales han enfatizado que el aprendizaje autoregulado es más que un proceso individual que involucra interacciones sociales ${ }^{27}$.

\section{Referencias}

1. Márquez C, Fasce E, Pérez C, Ortega J, Parra P, Ortiz L, et al. Aprendizaje autodirigido y su relación con estilos y estrategias de aprendizaje en estudiantes de medicina. Rev Med Chile 2014; 1422-30.

2. Dehnad A, Afsharianb F, Hosseinic F, Arabshahid SKS, Bigdelie S. Pursuing a definition of self-directed learning in literature from 2000-2012. Procedia Soc Behav Sci 2014; 116: 5184-7.

3. Jons-Cox L. The self-directed osteopathic medical student: Bringing adult learning into the osteopathic manipulative technique lab. Int J Osteopath Med 2014; 17: 61-5.

4. Kovalenko N, Yu A. Self-directed learning through creative activity of students International Conference on Research Paradigms Transformation in Social Sciences 2014. Procedia Soc Behav Sci 2015; 166: 393-8.

5. Embo M, Driessen E, Valcke M, Van Der Vleutene C. Assessment and feedback to facilitate self-directed learning in clinical practice of Midwifery students. Medical Teacher 2010; 32: e263-e9.

6. Deyo Z, Huynh D, Rochester C, Sturpe DA, Kiser K. Readiness for Self-directed Learning and Academic Performance in an Abilities Laboratory Course. Am J Pharm Educ 2011; 75 (2) Article 25: 1-6.

7. Dynan L, Cate T, Rhee K. The impact of learning structure on students' readiness for self-directed learning. Journal of Education for Business 2008; 84 (2): 96-100.
8. Fasce E, Pérez C, Ortiz L, Parra P, Matus O. Estructura factorial y confiabilidad de la escala de aprendizaje autodirigido de Fisher, King \& Tague en alumnos de medicina chilenos. Rev Med Chile 2011; 139: 1437-43.

9. Sporman C, Pérez C, Fasce, E, Ortega J, Bastías N, Bustamante C, Ibáñez P. Predictores afectivos y académicos del aprendizaje autodirigido en estudiantes de medicina. Rev Med Chile 2015; 143: 374-82.

10. Strauss A, Corbin J. Bases de la investigación cualitativa. Editorial Universidad de Antioquía. Medellín 2002.

11. Flick U. Introducción a la investigación cualitativa. Ediciones Morara S.L. Madrid 2004.

12. Vieytes R. Metodología de la investigación en organizaciones, mercado y sociedad. Editorial de las Ciencias. Buenos Aires 2005.

13. Daily J, Landis B. The journey top becoming an adult learner: from dependent to self-directed learning. J Am Coll Cardiol 2014; 64: 2066-8.

14. Moja L, Kwag K. Point of care information services: a platform for self-directed continuing medical education for front line decision makers. Postgrad Med J 2015; 91 : 83-91.

15. Murad M, Cato-Iglesias F, Varkey P, Prokp L, Murad A. The effectiveness of self-directed learning in health professions education: a systematic review. Med Educ 2010; 44: 1057-68.

16. Blomm BS. Effects of continuing medical education on improving physician clinical care and patiente health: a review of systematic reviews. Int J Technol Assess Gealth Care 2005; 21: 380-5.

17. Kar S, Premarajan K, Ramalingam A, Iswarya S, Sujiv A, Subitha L. Self-directed learning readiness among fifth semester MBBS students in a teaching institution of South India. Educ Health (Abingdon) 2014; 27: 289-92.

18. McGrath D, Cowley L, Rao S, Toomey M, Hannigan A, Murphy L, et al. Outcomes of Irish Graduate entry medical student engagement with self-directed learning of clinical skills. BMV Med Educ 2015; 15-21.

19. Prenkumar K, Pahwa P, Banerjee K, Baptiste K, Bhatt H, Lim H. Does medical training promote or deter self-directed learning? A longitudinal mixed-lethods study. Acad Med 2013; 88: 1754-64.

20. Gade S, Chari S. Case-based learning in endocrine physiology: an approach toward self-directed learning and the development of soft skills in medical students. Adv Physiol Educ 2013; 37: 356-60.

21. Tolsgaard M, Arendrup H, Podersen P, Ringsted C. Feasibility of self-directed learning in clerkship. Med Teach 2013; 35: e1409-15.

22. Van Schaik S, Plant J, O`Sullivan P. Promoting self-directed learning through portfolios in undergraduate 
medical education: The mentor`s perspective. Med Teach 2013; 35: 139-44.

23. Khabaz M, Aghili R, Emani Z, Malek M, Baradarn H, Taghavinia M, et al. Study guides: effective tools to improve self-directed learning skills of medical students. Acta Med Iran 2014: 52: 781-5.

24. Mahmud W, Haroon M, Munir A, Hyder O. Self-directed learning and research attitudes among medical students. J Coll Physicians Surg Pak 2014; 24: 173-7.
25. El-Gilany AH, Abusaad FES. Self-direct learning readiness and learning styles among Saudi undergraduate nursing students. Nurse Education Today 2013; 33 (9): 1040-4.

26. Garrison DR. Self-directed learning: toward a comprehensive model. Adult Educ Q 1997; 48 (1): 18.

27. Alvi E, Gillies R. Social interactions that support students'self-regulated learning: A case study of one teacher's experiences. Int J Educ Res 2015; 72: 14-25. 